Addendum

p53 regulates epithelialmesenchymal transition and stem cell properties through modulating miRNAs

\author{
Chun-Ju Chang, Chi-Hong Chao, Weiya Xia, Jer-Yen \\ Yang, Yan Xiong, Chia-Wei Li, Wen-Hsuan Yu, Sumaiyah \\ K. Rehman, Jennifer L. Hsu, Heng-Huan Lee, Mo Liu, \\ Chun-Te Chen, Dihua Yu and Mien-Chie Hung
}

Nat. Cell Biol. 13, 317-323 (2011); published online 20 February 2011

ZEB1 is a protein of 1124 amino acids with a predicted relative molecular mass $\sim 124 \mathrm{~K}$. However, in Supplementary Fig. S5 (corresponding to Fig. 4a) of our manuscript, the uncropped image of the ZEB1 immunoblot shows two bands with a relative molecular mass of $200 \mathrm{~K}$ and $125 \mathrm{~K}$, respectively. Two major ZEB1 signals $\left(M_{\mathrm{r}} 124 \mathrm{~K}\right.$ and $\left.200 \mathrm{~K}\right)$ have been observed in western blots when using different antibodies in various cell types (please see references 1-4 below). Therefore, to examine whether the bands observed in our study represent ZEB1, we knocked down ZEB1 in MCF12A control and p53 ${ }^{\mathrm{R} 175 \mathrm{H}}$ mutant cells using shRNA (Fig. 1a) and siRNA (Fig. 1b). As shown in the figure to the right, both of the bands are diminished by knockdown of ZEB1, indicating that these bands are ZEB1. siRNA: SASI_Hs02_00330526: GTTTGAAAAGATGCAAGCT; SASI_Hs02_00330527: GTGGCCCATTACAGGCAAC shRNA: Hairpin sequence for TRCN0000017566

1. Gregory, P. A. et al. Nat. Cell Biol. 10, 593-601 (2008).

2. Wellner, U. et al. Nat. Cell Biol. 11, 1487-1495 (2009).

3. Pantuck A. J. et al. Cancer Res. 70, 752-761 (2010).

4. Wang, F. et al. Cancer Res. 67, 8486-8493 (2007).
(A) $z$

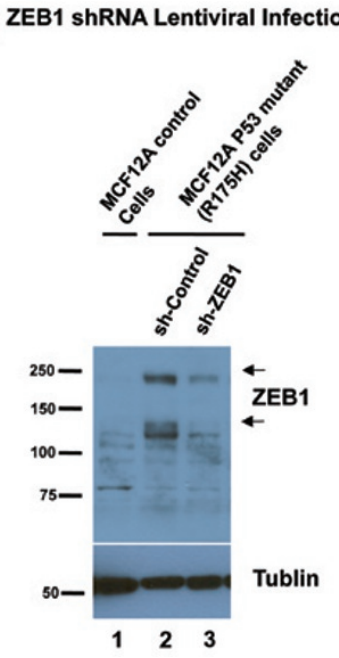

(B) ZEB1 siRNA Transfection

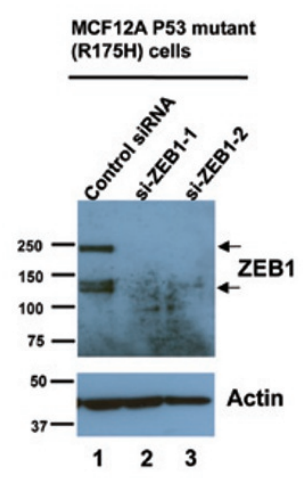

Figure 1 Downregulation of ZEB1 expression by shRNA or siRNAs in MCF12A control and $p 53^{\mathrm{R} 175 \mathrm{H}}$ mutant cells. (a) MCF $12 \mathrm{~A}$ cells expressing $\mathrm{p} 53^{\mathrm{R} 175 \mathrm{H}}$ mutant were infected with lentivirus encoding control shRNA or ZEB1 shRNA (clone ID: TRCN0000017566, Open Biosystems). After puromycine selection for 10 days, infected cells were subjected to immunoblotting analysis (ZEB1 (D80D3) antibody, \#3396, Cell signalling; 1:1000). Immunoblot shows the expression of ZEB1 from MCF12A control and infected p53 ${ }^{\mathrm{R} 175 \mathrm{H}}$ cells. (b) MCF12A cells expressing $p 53^{R 175 H}$ were transfected with two individual ZEB1 siRNAs (ZEB1-1, Iane2, SASI_HsO2_00330526, Sigma; ZEB1-2, SASI_Hs02_00330527, Sigma). At 48-h post-transfection, transfected cells were subjected to immunoblotting analysis.

\title{
Corrigendum
}

\section{Midbody accumulation through evasion of autophagy contributes to cellular reprogramming and tumorigenicity}

\author{
Tse-Chun Kuo, Chun-Ting Chen, Desiree Baron, Tamer T. Onder, Sabine Loewer, Sandra Almeida, Cara M.
} Weismann, Ping Xu, Jean-Marie Houghton, Fen-Biao Gao, George Q. Daley and Stephen Doxsey

Nat. Cell Biol. 13, 1214-1223 (2011); published online 11 September 2011; corrected after print 15 November 2011

In the version of this article initially published online and in print, the first sentence in the Acknowledgements section was incorrect. The correct sentence is:

We thank E. Baehrecke for critical reading of the manuscript, T. Schlaeger and colleagues for assistance with H1-OGN and associated cell lines, the University of Massachusetts Medical School (UMMS) Flow Facility for assistance with MBd enrichment, P. Furcinitti of UMMS Digital Light Microscopy Core Facility for assistance with imaging, the UMMS DERC Morphology Core for assistance with immunohistochemitry, D. Guertin and C. Sparks for assistance with SMP preparation, S. Lyle and C. Powers for performing EM experiments and H-L. Liu for assistance with clone construction. This error has been corrected in the HTML and PDF versions of the article. 